RIMS-1711

ON THE VALUE-DISTRIBUTION OF

LOGARITHMIC DERIVATIVES OF

DIRICHLET L-FUNCTIONS

By

Yasutaka IHARA and Kohji MATSUMOTO

$\underline{\text { January } 2011}$

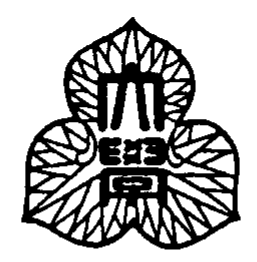

京都大学 数理解析研究所

RESEARCH INSTITUTE FOR MATHEMATICAL SCIENCES

KYOTO UNIVERSITY, Kyoto, Japan 


\title{
ON THE VALUE-DISTRIBUTION OF LOGARITHMIC DERIVATIVES OF DIRICHLET $L$-FUNCTIONS
}

\author{
YASUTAKA IHARA AND KOHJI MATSUMOTO
}

\begin{abstract}
We shall prove an unconditional basic result related to the value-distributions of $\left\{\left(L^{\prime} / L\right)(s, \chi)\right\}_{\chi}$ and of $\left\{\left(\zeta^{\prime} / \zeta\right)(s+i \tau)\right\}_{\tau}$, where $\chi$ runs over Dirichlet characters with prime conductors and $\tau$ runs over $\mathbf{R}$. The result asserts that the expected density function common for these distributions are in fact the density function in an appropriate sense. Under the Generalized Riemann hypothesis, stronger results have been proved in our previous articles, but our present result is unconditional.
\end{abstract}

\section{InTRODUCTION AND STATEMENT OF THE RESUlT}

This is a supplement of our former papers [3] and [7]. In [3], we defined and studied the "would-be density function" $M_{\sigma}(w)(\sigma>1 / 2)$ for the value-distribution of $L^{\prime} / L(s, \chi)$ on the complex plane $\mathbf{C}$ for certain family of $L$-functions over any global field ( $s$ : fixed with $\Re(s)=\sigma$ ), and established the expected connection under some restrictive hypothesis. This was generalized and strengthened in [8] under GRH, the Generalized Riemann hypothesis. In [7] we treated the analogous "would-be" density function $\mathcal{M}_{\sigma}(w)$ for the $\log L$ case, and in this case, when the base field is the rational number field $\mathbb{Q}$, we were able to obtain an unconditional result on the expected connection. The purpose of the present paper is to show that a parallel unconditional result for the $L^{\prime} / L$ case over $\mathbb{Q}$ can be obtained with but small modifications of the methods used in [7].

Let $s=\sigma+i \tau$ be a complex variable, $\zeta(s)$ be the Riemann zeta-function, $\chi$ a Dirichlet character with prime conductor, and $L(s, \chi)$ the associated Dirichlet $L$-function. We study the value-distribution of $\left(L^{\prime} / L\right)(s, \chi)$ when $\chi$ varies, or $\left(\zeta^{\prime} / \zeta\right)\left(s+i \tau^{\prime}\right)$ when $\tau^{\prime}$ varies. In the latter case, defining $\chi_{\tau^{\prime}}(n)=n^{-i \tau^{\prime}}\left(\tau^{\prime} \in \mathbb{R}, n=1,2, \ldots\right)$, we may regard that $\zeta\left(s+i \tau^{\prime}\right)=L\left(s, \chi_{\tau^{\prime}}\right)$ and the "character" $\chi_{\tau^{\prime}}$ varies. Therefore our object consists of two types of infinite families of characters, (FI) all Dirichlet characters $\chi$ of prime conductors, or (FII) characters of the form $\chi_{\tau^{\prime}}, \tau^{\prime} \in \mathbb{R}$.

Let $M_{\sigma}(w)$ for $\sigma>1 / 2$ be the function of $w \in \mathbb{C}$ defined in [3]. Here we take $K=\mathbf{Q}$ (in terms of [8], this corresponds to the function $M_{\sigma}(w)$ for "Case 1 ", $\left.K=\mathbf{Q}, P_{\infty}=(\infty)\right)$.

We shall prove the following theorem.

Theorem 1.1. Let $s=\sigma+i \tau \in \mathbb{C}$ be fixed, with $\sigma=\Re s>1 / 2$. Then the equality

$$
\operatorname{Avg}_{\chi} \Phi\left(\frac{L^{\prime}}{L}(s, \chi)\right)=\int_{\mathbb{C}} M_{\sigma}(w) \Phi(w)|d w|
$$

2000 Mathematics Subject Classification. Primary 11M06.

Key words and phrases. Dirichlet $L$-function, Riemann zeta-function, value-distribution, density function. 
holds simultaneously for both families (FI) and (FII), where $|d w|=d u d v / 2 \pi$ for $w=u+i v$, the meaning of $\operatorname{Avg}_{\chi}$ is defined below, and the test function $\Phi$ is one of the following:

(i) $\Phi$ is any continuous bounded function;

(ii) $\Phi$ is the characteristic function of either a compact subset of $\mathbb{C}$ or the complement of such a subset.

Finally, when $s=1$, (at least) in case of the family (FI), the test function $\Phi$ can be any continuous function of at most polynomial growth.

The above statement for $\sigma>1$, and stronger but conditional results for $\sigma>1 / 2$ under GRH (over more general base fields for the family (FI)) were already shown in $[3,8]$ (cf. also a survey article [5]). The purpose of the present paper is to prove this theorem unconditionally for any $\sigma>1 / 2$.

The definition of $\operatorname{Avg}_{\chi}$ is as follows.

Case (FI). For any prime $f(>2)$, let $X(f)$ denote the set of all primitive Dirichlet characters whose conductor is precisely $f$, and $X^{\prime}(f)=X^{\prime}(f, s)$ be the subset of $X(f)$ consisting of all $\chi$ such that $L(s, \chi) \neq 0$ for our fixed $s$. By a theorem of Montgomery [14] it satisfies

$$
\lim _{f \rightarrow \infty} \frac{\left|X^{\prime}(f)\right|}{|X(f)|}=1
$$

(For any finite set $A$ we denote by $|A|$ its cardinality.) For any complex-valued function $\phi(\chi)$ on $X^{\prime}(f)$, we define the averages

$$
\begin{gathered}
\operatorname{Avg}_{X^{\prime}(f)} \phi(\chi)=\frac{1}{|X(f)|} \sum_{\chi \in X^{\prime}(f)} \phi(\chi), \\
\operatorname{Avg}_{f \leq m} \phi(\chi)=\frac{1}{\pi(m)} \sum_{f \leq m} \operatorname{Avg}_{X^{\prime}(f)} \phi(\chi),
\end{gathered}
$$

where $m$ is any positive integer, $f$ runs over all odd prime numbers up to $m$, and $\pi(m)$ denotes the number of prime numbers up to $m$. Now define

$$
\operatorname{Avg}_{\chi} \phi(\chi)=\lim _{m \rightarrow \infty}\left(\operatorname{Avg}_{f \leq m} \phi(\chi)\right) .
$$

When we state a formula for $\operatorname{Avg}_{\chi}$, it will always include the claim that the limit exists. We remark here that the main statement of the theorem deals only with the averages of those $\phi(\chi)$ which are bounded on the union of $X^{\prime}(f)$ over all $f$ (because the test function $\Phi$ is bounded). Therefore, if we replace $X^{\prime}(f)$ by a smaller subset preserving the condition (1.2), the average (1.3) (resp. (1.4)) changes only by a quantity which tends to 0 as $f \rightarrow \infty$ (resp. $m \rightarrow \infty$ ), hence the limit average (1.5) remains the same (e.g. the subset " $X^{\prime}(f)$ " in [7], or the subset denoted by $X^{\prime \prime}(f)$ defined below in Section 2 used for the proof). As regards the additional statement for $s=1$, note that $X^{\prime}(f, 1)=X(f)$.

Case (FII). The definition of $\operatorname{Avg}_{\chi}$ in this case is simply

$$
\operatorname{Avg}_{\chi} \phi\left(\chi_{\tau^{\prime}}\right)=\lim _{T \rightarrow \infty} \frac{1}{2 T} \int_{-T}^{T} \phi\left(\chi_{\tau^{\prime}}\right) d \tau^{\prime}
$$


for any integrable function $\phi\left(\chi_{\tau^{\prime}}\right)$ of $\tau^{\prime}$.

A closely related problem is the study on the value-distribution of $\log L(s, \chi)$. In [7], we have constructed a continuous non-negative density function $\mathcal{M}_{\sigma}(w)$ parametrized by $\sigma>1 / 2$ and established the following theorem.

Theorem 1.2. ([7]) For any $s \in \mathbb{C}$ with $\sigma=\Re(s)>1 / 2$,

$$
\operatorname{Avg}_{\chi} \Phi(\log L(s, \chi))=\int_{\mathbb{C}} \mathcal{M}_{\sigma}(w) \Phi(w)|d w|
$$

holds simultaneously for both families (FI) and (FII) for a suitable choice of the branch of the logarithm, a suitable definition of the average $\operatorname{Avg}_{\chi}$, where $\Phi$ is as in Theorem 1.1.

Our Theorem 1.1 implies that the exact analogue of Theorem 1.2 holds in the $L^{\prime} / L$ case.

To prove these unconditional results, our method is to apply several mean value results on $L$-functions. As for the $\log L$ case, such mean value theorems were obtained in [7] to prove Theorem 1.2. It is possible to use the same mean value theorems in our present situation, because $L^{\prime} / L$ can be written as an integral involving $\log L$ in the integrand, by using the Cauchy integral formula. Note that the idea of applying the Cauchy integral formula in such a situation already appeared in Kershner and Wintner [12] in the (FII) case (see Remark 3.1).

In the following sections we will prove Theorem 1.1. Since the basic structure of the proof is similar to those developed in [3] [7], we will only point out the differences from those and omit the details.

\section{Proof in the CASe (FI)}

As mentioned in Section 1, the assertion of Theorem 1.1 was already shown in [3] when $\sigma>1$. Therefore it is sufficient to consider the case $1 / 2<\sigma \leq 1$. The final statement for $s=1$ then follows directly by combining [9]§5 (Theorem 5) with [8]§5 Lemma A.

As in [7, Section 7], let $1 / 2<\sigma_{0}<1,0<3 \varepsilon_{1}<\sigma_{0}-1 / 2, \alpha_{0}=\sigma_{0}-\varepsilon_{1}, \alpha_{1}=\sigma_{0}-2 \varepsilon_{1}$, $\alpha_{2}=1 / 2+\varepsilon_{1}$. Then $1 / 2<\alpha_{2}<\alpha_{1}<\alpha_{0}<\sigma_{0}<1$. These constants are regarded to be fixed, and the implied constants of Landau's $O$-symbol or Vinogradov's symbol below may depend on them.

Let $T=|\tau|+2$, and let $X^{\prime \prime}(f)$ be the set of all $\chi \in X(f)$ for which $L\left(s^{\prime}, \chi\right) \neq 0$ for any $s^{\prime}=\sigma^{\prime}+i \tau^{\prime}$ in the region $\sigma^{\prime} \geq \sigma_{0},\left|\tau^{\prime}\right| \leq T$. Then obviously, $X^{\prime \prime}(f) \subset X^{\prime}(f)$ and Proposition 2.1 of [7] (which is based on a theorem of Montgomery [14]) asserts that

$$
\lim _{f \rightarrow \infty} \frac{\left|X^{\prime \prime}(f)\right|}{|X(f)|}=1
$$

So it suffices to prove the theorem where the average is defined with respect to $X^{\prime \prime}(f)$.

We study the case $\Phi=\psi_{z}$ first, where $z \in \mathbb{C}$ and $\psi_{z}$ is the additive character of $\mathbb{C}$ defined by $\psi_{z}(w)=\exp (i \Re(\bar{z} w))$. When once this case is established, we can deduce the 
assertion of the case (FI) of Theorem 1.1 for general $\Phi$ satisfying (i) and (ii), quite similarly to the argument in [7, Section 9] (see also Remark 3.2).

In the case $\Phi=\psi_{z}$, the right-hand side of (1.1) is equal to

$$
\int_{\mathbb{C}} M_{\sigma}(w) \psi_{z}(w)|d w|=\widetilde{M}_{\sigma}(z),
$$

the Fourier dual of $M_{\sigma}(z)$ (see Theorem 3 of [3]). Since $\psi_{z}$ is bounded, the average (1.3) (and so (1.4), (1.5)) does not change if we replace $X^{\prime}(f)$ by $X^{\prime \prime}(f)$. Therefore, noting $|X(f)|=f-2$ for any odd prime $f$, we find that what we have to prove in this case is

$$
\lim _{m \rightarrow \infty} \frac{1}{\pi(m)} \sum_{f \leq m} \frac{1}{f-2} \sum_{\chi \in X^{\prime \prime}(f)} \psi_{z}\left(\frac{L^{\prime}}{L}(s, \chi)\right)=\widetilde{M}_{\sigma}(z) .
$$

First we introduce the "finite truncation" of $L$-functions. Let $1<y<m, P=P_{y}$ the set of all primes not greater than $y$, and write $P=\left\{p_{1}, \ldots, p_{r}\right\}, r=\pi(y) \sim y / \log y$. Define

$$
L_{P}(s, \chi)=\prod_{p \in P}\left(1-\chi(p) p^{-s}\right)^{-1}
$$

and

$$
\log L_{P}(s, \chi)=-\sum_{p \in P} \log \left(1-\chi(p) p^{-s}\right),
$$

where "Log" means the principal branch. As in [3], let $M_{\sigma, P}(w)$ be the density function for the value-distribution of $\left(L_{P}^{\prime} / L_{P}\right)(s, \chi)$, and $\widetilde{M}_{\sigma, P}(z)$ be its Fourier dual.

The starting point of the proof of (2.2) is the following inequality:

$$
\begin{aligned}
& \left|\frac{1}{\pi(m)} \sum_{f \leq m} \frac{1}{f-2} \sum_{\chi \in X^{\prime \prime}(f)} \psi_{z}\left(\frac{L^{\prime}}{L}(s, \chi)\right)-\widetilde{M}_{\sigma}(z)\right| \\
& \leq\left|\frac{1}{\pi(m)} \sum_{f \leq m} \frac{1}{f-2} \sum_{\chi \in X^{\prime \prime}(f)}\left\{\psi_{z}\left(\frac{L^{\prime}}{L}(s, \chi)\right)-\psi_{z}\left(\frac{L_{P}^{\prime}}{L_{P}}(s, \chi)\right)\right\}\right| \\
& +\left|\frac{1}{\pi(m)} \sum_{f \leq m} \frac{1}{f-2} \sum_{\chi \in X^{\prime \prime}(f)} \psi_{z}\left(\frac{L_{P}^{\prime}}{L_{P}}(s, \chi)\right)-\widetilde{M}_{\sigma, P}(z)\right| \\
& +\left|\widetilde{M}_{\sigma, P}(z)-\widetilde{M}_{\sigma}(z)\right| \\
& =X_{P}^{l d}(z)+Y_{P}^{l d}(z)+Z_{P}^{l d}(z)
\end{aligned}
$$

say. This is an analogue of $[7,(125)]$, and "ld"s (which stand for the "logarithmic derivative") are attached only for the purpose of distinguishing our notation from that in [7].

In order to estimate $X_{P}^{l d}(z)$, we first introduce some more notation. For each Dirichlet character $\chi$, from the half-plane $\left\{s^{\prime} \mid \sigma^{\prime}>1 / 2\right\}$ we exclude all the segments of the form $\left\{\sigma^{\prime}+i \Im \rho \mid 1 / 2<\sigma^{\prime} \leq \Re \rho\right\}$ (for all possible zeros $\rho$ of $L\left(s^{\prime}, \chi\right.$ ) with $\Re \rho>1 / 2$ ), and denote the remaining region by $G_{\chi}$. In the region $G_{\chi}$, we can define the value of $\log L\left(s^{\prime}, \chi\right)$ by 
the analytic continuation along the horizontal path $\left\{\sigma^{\prime \prime}+i \tau^{\prime} \mid \sigma^{\prime \prime} \geq \sigma^{\prime}\right\}$. Define

$$
R_{P}\left(s^{\prime}, \chi\right)=\log L\left(s^{\prime}, \chi\right)-\log L_{P}\left(s^{\prime}, \chi\right)
$$

for $s^{\prime} \in G_{\chi}\left(\alpha_{1}\right)=G_{\chi} \cap\left\{\sigma^{\prime}>\alpha_{1}\right\}$. Let $c$ and $\delta$ be fixed small positive numbers, and let $\beta_{0}=\beta_{0}(\delta)>1, \beta_{1}=\beta_{1}(\delta)=2 \beta_{0}, H(\tau), Q_{0}(\tau), Q_{1}(\tau), f_{P}\left(s^{\prime}, \chi\right), F_{P}(\tau, \chi)$ be as in [7, Section 7]. The distance between the boundaries of the two sets $Q_{0}(\tau)$ and $Q_{1}(\tau)$ is $\varepsilon_{2}=\min \left\{\varepsilon_{1}, c\right\}$. Let $X_{1}(f)$ be the set of all $\chi \in X^{\prime \prime}(f)$ such that

$$
F_{P}(\tau, \chi) \geq \pi\left(\frac{\varepsilon_{2}}{2}\right)^{2}\left(\frac{\delta}{2}\right)^{2}
$$

and $X_{2}(f)$ its complement in $X^{\prime \prime}(f)$, that is, all those $\chi \in X^{\prime \prime}(f)$ satisfying

$$
F_{P}(\tau, \chi)<\pi\left(\frac{\varepsilon_{2}}{2}\right)^{2}\left(\frac{\delta}{2}\right)^{2}
$$

We divide

$$
\sum_{\chi \in X^{\prime \prime}(f)}\left\{\psi_{z}\left(\frac{L^{\prime}}{L}(s, \chi)\right)-\psi_{z}\left(\frac{L_{P}^{\prime}}{L_{P}}(s, \chi)\right)\right\}=\sum_{\chi \in X_{1}(f)}+\sum_{\chi \in X_{2}(f)}=S_{1}^{l d}(f)+S_{2}^{l d}(f),
$$

say.

Consider $S_{2}^{l d}(f)$. First, using the fact $\left|\psi_{z}(w)-\psi_{z}\left(w^{\prime}\right)\right| \leq|z| \cdot\left|w-w^{\prime}\right|([3,(6.5 .19)])$, we obtain

$$
\left|S_{2}^{l d}(f)\right| \leq|z| \sum_{\chi \in X_{2}(f)}\left|\frac{L^{\prime}}{L}(s, \chi)-\frac{L_{P}^{\prime}}{L_{P}}(s, \chi)\right| .
$$

Since (2.5) holds for $\chi \in X_{2}(f)$, by Lemma 7.2 of [7] we obtain

$$
\left|f_{P}\left(s^{\prime}, \chi\right)\right|<\delta / 2 \quad\left(s^{\prime} \in Q_{0}(\tau)\right) .
$$

Therefore by Lemma 7.1 of [7] we find that $H(\tau) \subset G_{\chi}\left(\alpha_{1}\right)$ (especially $L\left(s^{\prime}, \chi\right) \neq 0$ for $\left.s^{\prime} \in H(\tau)\right)$, and $\left|R_{P}\left(s^{\prime}, \chi\right)\right|<\delta$ for $s^{\prime} \in H(\tau)$.

Let $U=U(s)$ be the circle of radius $\varepsilon_{2} / 2$ whose center is $s$. Then $U \subset H(\tau)$ (because $\left.\sigma-\varepsilon_{2} / 2 \geq \sigma_{0}-\varepsilon_{2} / 2>\sigma_{0}-\varepsilon_{1}=\alpha_{0}\right)$, and so $\left(L^{\prime} / L\right)\left(s^{\prime}, \chi\right)$ is holomorphic on and inside $U$. Therefore by the Cauchy integral formula we have

$$
\begin{aligned}
\frac{L^{\prime}}{L}(s, \chi) & =(\log L(s, \chi))^{\prime}=\frac{1}{2 \pi i} \int_{U(s)} \frac{\log L\left(s^{\prime}, \chi\right)}{\left(s^{\prime}-s\right)^{2}} d s^{\prime} \\
& =\frac{1}{\pi \varepsilon_{2}} \int_{0}^{2 \pi} \log L\left(s+\frac{\varepsilon_{2}}{2} e^{i \theta}, \chi\right) e^{-i \theta} d \theta,
\end{aligned}
$$

and similarly

$$
\frac{L_{P}^{\prime}}{L_{P}}(s, \chi)=\frac{1}{\pi \varepsilon_{2}} \int_{0}^{2 \pi} \log L_{P}\left(s+\frac{\varepsilon_{2}}{2} e^{i \theta}, \chi\right) e^{-i \theta} d \theta .
$$

Substituting (2.9) and (2.10) into (2.7), we obtain

$$
\left|S_{2}^{l d}(f)\right| \leq \frac{|z|}{\pi \varepsilon_{2}} \int_{0}^{2 \pi} \sum_{\chi \in X_{2}(f)}\left|R_{P}\left(s+\frac{\varepsilon_{2}}{2} e^{i \theta}, \chi\right)\right| d \theta .
$$


Here we note that $U \subset Q_{0}(\tau)$. In fact, we have already seen that $U \subset H(\tau)$, and also we see $U \subset\left\{\sigma^{\prime}<\beta_{0}\right\}$ because $\beta_{0}$ is large. Therefore (2.8) holds for $s^{\prime} \in U$. This implies, as is shown in the proof of Lemma 7.1 of [7],

$$
\left|R_{P}\left(s^{\prime}, \chi\right)\right| \leq 2\left|f_{P}\left(s^{\prime}, \chi\right)\right| \quad\left(s^{\prime} \in U\right) .
$$

Combining (2.11) and (2.12), and using Schwarz' inequality, we have

$$
\begin{aligned}
\left|S_{2}^{l d}(f)\right| & \leq \frac{2|z|}{\pi \varepsilon_{2}} \int_{0}^{2 \pi} \sum_{\chi \in X_{2}(f)}\left|f_{P}\left(s+\frac{\varepsilon_{2}}{2} e^{i \theta}, \chi\right)\right| d \theta \\
& \ll|z| f^{1 / 2} \int_{0}^{2 \pi}\left(\sum_{\chi \in X_{2}(f)}\left|f_{P}\left(s+\frac{\varepsilon_{2}}{2} e^{i \theta}, \chi\right)\right|^{2}\right)^{1 / 2} d \theta .
\end{aligned}
$$

Since $\sigma^{\prime}=\Re\left(s+\left(\varepsilon_{2} / 2\right) e^{i \theta}\right)>\alpha_{0}>\alpha_{1}$ for $s^{\prime}=\sigma^{\prime}+i \tau^{\prime} \in U$, using [7, (133)] (this is the point where a mean-value result on $L$-functions is necessary) we obtain

$$
\left|S_{2}^{l d}(f)\right| \ll|z| f^{1 / 2} A\left(\tau^{\prime}, f, y\right)^{1 / 2} \ll|z| f^{1 / 2} A(\tau, f, y)^{1 / 2},
$$

where

$$
A(\tau, f, y)=f y^{1-2 \alpha_{1}}+f^{\left(1-\alpha_{1}\right) /\left(1-\alpha_{2}\right)} \exp \left(B_{0} \frac{y^{1-\alpha_{2}}}{\log y}\right)\left(1+\frac{|\tau|+1}{f^{2 \alpha_{2}}}\right)
$$

with a certain absolute positive constant $B_{0}$.

The treatment of $S_{1}^{l d}(f)$ can be done exactly in the same manner as in the argument around [7, (135), (136)]. We have $\left|S_{1}^{l d}(f)\right| \ll A(\tau, f, y)$, and, combining this with (2.14), we obtain

$$
X_{P}^{l d}(z) \ll \frac{1}{\pi(m)} \sum_{f \leq m} \frac{1}{f}\left(|z| f^{1 / 2} A(\tau, f, y)^{1 / 2}+A(\tau, f, y)\right) .
$$

This is the $L^{\prime} / L$-analogue (exactly the same form!) of Proposition 7.4 of [7].

Now we consider $Y_{P}^{l d}(z)$. Divide

$$
\frac{1}{\pi(m)} \sum_{f \leq m} \frac{1}{f-2} \sum_{\chi \in X^{\prime \prime}(f)} \psi_{z}\left(\frac{L_{P}^{\prime}}{L_{P}}(s, \chi)\right)
$$

into $J_{0}^{l d(m)}+J_{1}^{l d(m)}+J_{2}^{l d(m)}$, analogously to the decomposition of $[7,(137)]$. The treatment of $J_{0}^{l d(m)}$ and $J_{2}^{l d(m)}$ is exactly the same as that of $J_{0}^{(m)}$ and $J_{2}^{(m)}$ in [7]. As for $J_{1}^{l d(m)}$, we first note that, when the conductor $f$ of $\chi$ is larger than $y$, it holds that

$$
\psi_{z}\left(\frac{L_{P}^{\prime}}{L_{P}}(s, \chi)\right)=\sum_{\mathbf{n}_{P} \in \mathbb{Z}_{P}} A_{\sigma, P}^{l d}\left(\mathbf{n}_{P} ; z, \bar{z}\right) \chi_{P}^{\mathbf{n}_{P}} P^{-i \tau \mathbf{n}_{P}},
$$

where $\mathbb{Z}_{P}=\prod_{p \in P} \mathbb{Z}$, and for $\mathbf{n}_{P}=\left(n_{p}\right)_{p \in P} \in \mathbb{Z}_{P}$,

$$
\chi_{P}^{\mathbf{n}_{P}}=\prod_{p \in P} \chi(p)^{n_{p}}, \quad P^{-i \tau \mathbf{n}_{P}}=\prod_{p \in P} p^{-i \tau n_{p}}
$$

and $A_{\sigma, P}^{l d}\left(\mathbf{n}_{P} ; z, \bar{z}\right)$ is given by $[3,(5.1 .7)]$ (without "ld"). This follows from $[3,(1.5 .4)$, (5.1.6)], and is the $L^{\prime} / L$-analogue of [7, (138)]. Starting from (2.17), we proceed similarly to the argument around $[7,(139)-(147)]$. (On this occasion we note that $\sum_{n_{p} \in \mathbb{Z}}$ is missing 
after the product symbol $\prod_{p \in P}$ in the first line of $[7,(147)]$.) We use $[3,(5.1 .14)]$ instead of $[7,(89)]$, and $[3,(3.1 .10)]$ instead of $[7,(32)]$. Proposition 5.3 of [7] includes the present $L^{\prime} / L$ case, and so we can apply it. Then, instead of $\eta(y)$ in $[7]$ (see $[7,(116)]$ ),

$$
\eta^{l d}(y)=\eta^{l d}(\sigma, y)=\left\{\begin{array}{lll}
y^{1-\sigma} & \text { if } & 1 / 2<\sigma<1 \\
\log y & \text { if } & \sigma=1
\end{array}\right.
$$

appears. The conclusion is that $Y_{P}^{l d}(z)$ satisfies the same inequality as that in Proposition 7.5 of [7] (with replacing $\eta(y)$ by $\eta^{l d}(y)$ ).

Finally we choose $y=(\log m)^{\omega_{2}}$ with $0<\omega_{2}<2$. Then we find that $X_{P}^{l d}(z), Y_{P}^{l d}(z)$ tend to 0 as $m \rightarrow \infty$. Also Theorem 3 of [3] implies that $Z_{P}^{l d}(z) \rightarrow 0$ as $m \rightarrow \infty$. Therefore we now complete the proof of (2.2). Moreover this convergence is uniform in $|z| \leq R$ for any $R>0$.

\section{Proof in the Case (FII)}

As in the case (FI), it is enough to consider the case $\Phi=\psi_{z}$ i.e., to prove

$$
\lim _{T \rightarrow \infty} \frac{1}{2 T} \int_{-T}^{T} \psi_{z}\left(\frac{\zeta^{\prime}}{\zeta}\left(\sigma+i \tau^{\prime}\right)\right) d \tau^{\prime}=\widetilde{M}_{\sigma}(z)
$$

(cf. $[7,(92)]$ ). Similarly to $[7,(95)]$, we begin with the inequality

$$
\begin{aligned}
& \left|\frac{1}{2 T} \int_{-T}^{T} \psi_{z}\left(\frac{\zeta^{\prime}}{\zeta}\left(\sigma+i \tau^{\prime}\right)\right) d \tau^{\prime}-\widetilde{M}_{\sigma}(z)\right| \\
& \leq\left|\frac{1}{2 T} \int_{-T}^{T}\left\{\psi_{z}\left(\frac{\zeta^{\prime}}{\zeta}\left(\sigma+i \tau^{\prime}\right)\right)-\psi_{z}\left(\frac{\zeta_{P}^{\prime}}{\zeta_{P}}\left(\sigma+i \tau^{\prime}\right)\right)\right\} d \tau^{\prime}\right| \\
& \quad+\left|\frac{1}{2 T} \int_{-T}^{T} \psi_{z}\left(\frac{\zeta_{P}^{\prime}}{\zeta_{P}}\left(\sigma+i \tau^{\prime}\right)\right) d \tau^{\prime}-\widetilde{M}_{\sigma, P}(z)\right| \\
& +\left|\widetilde{M}_{\sigma, P}(z)-\widetilde{M}_{\sigma}(z)\right| \\
& =X_{P}^{l d}(z)+Y_{P}^{l d}(z)+Z_{P}^{l d}(z),
\end{aligned}
$$

say. Note that the meaning of these $X_{P}^{l d}(z), Y_{P}^{l d}(z), Z_{P}^{l d}(z)$ is different from that in Section 2 .

The method of evaluating $X_{P}^{l d}(z)$ is a little different from the argument in [7]; rather, we follow the idea in Section 2. Noting $\left|\psi_{z}\right|=1$ we have

$$
X_{P}^{l d}(z) \leq \frac{1}{2 T} \int_{-2}^{2} 2 d \tau^{\prime}+\frac{1}{2 T} \int_{I(T)}\left|\psi_{z}\left(\frac{\zeta^{\prime}}{\zeta}\left(\sigma+i \tau^{\prime}\right)\right)-\psi_{z}\left(\frac{\zeta_{P}^{\prime}}{\zeta_{P}}\left(\sigma+i \tau^{\prime}\right)\right)\right| d \tau^{\prime},
$$

where $I(T)=[-T,-2] \cup[2, T]$. Let $I_{1}(T)$ (resp. $I_{2}(T)$ ) be the set of all $\tau^{\prime} \in I(T)$ for which (2.4) (resp. (2.5)), with replacing $\tau$ by $\tau^{\prime}$ and putting $\chi=\mathbf{1}$ (the trivial character), holds. Decompose the second integral on the right-hand side of (3.3) as $X_{1}^{l d}+X_{2}^{l d}$, where $X_{j}^{l d}$ denotes the integral on $I_{j}(T)(j=1,2)$. Then

$$
X_{P}^{l d}(z) \leq \frac{4}{T}+\frac{1}{2 T}\left(X_{1}^{l d}+X_{2}^{l d}\right) .
$$


Consider $X_{2}^{l d}$. When $\tau^{\prime} \in I_{2}(T)$, as in Section 2 we see that $\zeta\left(s^{\prime \prime}\right) \neq 0$ and $\left|R_{P}\left(s^{\prime \prime}, \mathbf{1}\right)\right| \leq$ $2\left|f_{P}\left(s^{\prime \prime}, \mathbf{1}\right)\right|<\delta$ for any $s^{\prime \prime} \in H\left(\tau^{\prime}\right)$. Therefore $\left(\zeta^{\prime} / \zeta\right)\left(s^{\prime \prime}\right)$ is holomorphic on and inside the circle $U^{\prime}$ of radius $\varepsilon_{2} / 2$ whose center is $\sigma+i \tau^{\prime}$, so

$$
\frac{\zeta^{\prime}}{\zeta}\left(\sigma+i \tau^{\prime}\right)=\frac{1}{2 \pi i} \int_{U^{\prime}} \frac{\log \zeta\left(s^{\prime \prime}\right)}{\left(s^{\prime \prime}-\sigma-i \tau^{\prime}\right)^{2}} d s^{\prime \prime} .
$$

Similarly to (2.13), we obtain

$$
X_{2}^{l d} \ll|z| T^{1 / 2} \int_{0}^{2 \pi}\left(\int_{I_{2}(T)}\left|f_{P}\left(\sigma+i \tau^{\prime}+\frac{\varepsilon_{2}}{2} e^{i \theta}, \mathbf{1}\right)\right|^{2} d \tau^{\prime}\right)^{1 / 2} d \theta .
$$

A mean square estimate of $\left|f_{P}\right|$ was obtained in Lemma 5 of [13] (see also [7, (102), (106)]). Applying this lemma, we have

$$
\frac{1}{2 T} X_{2}^{l d} \ll|z|\left\{y^{1-2 \alpha_{1}+\varepsilon}+T^{1-2 \alpha_{1}+\varepsilon} \exp \left(C_{1}\left(\frac{y}{\log y}\right)^{1 / 2}\right)\right\},
$$

for any small $\varepsilon>0$ and an absolute constant $C_{1}>0$.

As for $X_{1}^{l d}$, we first use $\left|\psi_{z}\right|=1$ to obtain

$$
X_{1}^{l d} \leq 2 \operatorname{meas}\left(I_{1}(T)\right),
$$

where meas $(A)$ means the 1-dimensional Lebesgue measure of the set $A$. Using (2.4) for $\tau^{\prime} \in I_{1}(T)$, we have

$$
\begin{aligned}
\operatorname{meas}\left(I_{1}(T)\right) & \ll \int_{I_{1}(T)} F_{P}\left(\tau^{\prime}, \mathbf{1}\right) d \tau^{\prime} \\
& =\int_{\alpha_{1}}^{\beta_{1}} d \sigma^{\prime \prime} \int_{-T-2 c}^{T+2 c}\left|f_{P}\left(\sigma^{\prime \prime}+i \tau^{\prime \prime}, \mathbf{1}\right)\right|^{2} d \tau^{\prime \prime} \int_{J_{1}\left(\tau^{\prime \prime}\right)} d \tau^{\prime},
\end{aligned}
$$

where $J_{1}\left(\tau^{\prime \prime}\right)=I_{1}(T) \cap\left[\tau^{\prime \prime}-2 c, \tau^{\prime \prime}+2 c\right]$. The innermost integral is $\leq 4 c$, and is equal to 0 if $\tau^{\prime \prime} \in(-2+2 c, 2-2 c)$. Therefore we can apply Lemma 5 of [13] ([7, (102), (106)]) to the right-hand side of (3.9). Combining with (3.8), we obtain

$$
\begin{aligned}
\frac{1}{2 T} X_{1}^{l d} \ll & \int_{\alpha_{1}}^{2}\left\{y^{1-2 \alpha_{1}+\varepsilon}+T^{1-2 \alpha_{1}+\varepsilon} \exp \left(C_{1}\left(\frac{y}{\log y}\right)^{1 / 2}\right)\right\} d \sigma^{\prime \prime} \\
& +\int_{2}^{\beta_{1}}\left\{\frac{1}{\sigma^{\prime \prime}} y^{1-2 \sigma^{\prime \prime}+\varepsilon}+\frac{1}{\sigma^{\prime \prime} T} y^{2-2 \sigma^{\prime \prime}+\varepsilon}\right\} d \sigma^{\prime \prime} \\
\ll & y^{1-2 \alpha_{1}+\varepsilon}+T^{1-2 \alpha_{1}+\varepsilon} \exp \left(C_{1}\left(\frac{y}{\log y}\right)^{1 / 2}\right) \\
& +y^{-3+\varepsilon} \log \beta_{1}+\frac{1}{T} y^{-2+\varepsilon} \log \beta_{1} .
\end{aligned}
$$

Since the factor $\log \beta_{1}$ can be absorbed into the implied constant, from (3.4), (3.7) and (3.10) we obtain

$$
X_{P}^{l d}(z) \ll(|z|+1)\left\{y^{1-2 \alpha_{1}+\varepsilon}+T^{1-2 \alpha_{1}+\varepsilon} \exp \left(C_{1}\left(\frac{y}{\log y}\right)^{1 / 2}\right)\right\}+\frac{1}{T}+y^{-3+\varepsilon}
$$


The way of evaluating $Y_{P}^{l d}(z)$ is almost the same as that around [7, (109)—(122)]; only replace $\eta(y)$ by $\eta^{l d}(y)$. As an analogue of Proposition 6.2 of [7], we obtain

$$
Y_{P}^{l d}(z) \ll \frac{1}{T} \exp \left(C_{3}\left(|z| y^{3 / 2-\sigma}+\frac{y}{\log y}\right)\right)
$$

with an absolute constant $C_{3}>0$.

Choosing $y=(\log T)^{\omega_{1}}\left(0<\omega_{1}<1\right)$, from (3.11), (3.12) and Theorem 3 of [3] we find, as in [7], that $X_{P}^{l d}(z), Y_{P}^{l d}(z)$ and $Z_{P}^{l d}(z)$ tend to 0 as $T \rightarrow \infty$, uniformly in $|z| \leq R$ for any $R>0$. This proves (3.1).

Remark 3.1. Bohr and Jessen [2] proved the case (FII) of Theorem 1.2 for $\Phi$ with (ii), and Jessen and Wintner [10] reformulated the result in terms of asymptotic distribution functions. Kershner and Wintner [12] then proved that the analogue of the Jessen-Wintner theory is valid in the $\zeta^{\prime} / \zeta(s)$ case. Therefore the case (FII) of our Theorem 1.1, for $\Phi$ with (ii), is essentially included in Kershner and Wintner [12], though the density function is not explicitly given in their paper. The general (FII) case can be deduced from their result by the argument suggested in Remark 9.1 of [7]. Our method in the present paper is rather different from theirs, and has advantages such as the unified treatment of both the cases (FI) and (FII), and the explicit construction of the density function $M_{\sigma}(w)$. In fact, the function $M_{\sigma}(w)$ and its Fourier dual themselves are interesting objects of research (see [8], [4]).

Remark 3.2. To show the general conclusion of our theorem from the special case $\Phi=\psi_{z}$, we can apply the method given in [7, Section 9], as indicated at the beginning of Section 2. This step can be explained as a consequence of a general theorem on weak convergence of probability measures.

Here we show how to deduce the case (i) of Theorem 1.1 from the case $\Phi=\psi_{z}$. In case (FI), the left-hand side of (1.1) is

$$
\begin{aligned}
\lim _{m \rightarrow \infty} & \frac{1}{\pi(m)} \sum_{f \leq m} \frac{1}{X(f)} \sum_{\chi \in X^{\prime}(f)} \Phi\left(\frac{L^{\prime}}{L}(s, \chi)\right) \\
& =\lim _{m \rightarrow \infty} \frac{1}{\pi(m)-1} \sum_{f \leq m} \frac{1}{X^{\prime}(f)} \sum_{\chi \in X^{\prime}(f)} \Phi\left(\frac{L^{\prime}}{L}(s, \chi)\right) .
\end{aligned}
$$

Let $\delta_{w}$ be the complex Dirac measure which is non-zero only at $w$, and define

$$
\mu_{m}=\frac{1}{\pi(m)-1} \sum_{f \leq m} \frac{1}{X^{\prime}(f)} \sum_{\chi \in X^{\prime}(f)} \delta_{L^{\prime} / L(s, \chi)} .
$$

Then this is a probability measure, and the right-hand side of (3.13) can be written as

$$
\lim _{m \rightarrow \infty} \int_{\mathbb{C}} \Phi(w) d \mu_{m}(w) .
$$

Therefore (1.1) for any continuous bounded $\Phi$ is nothing but the weak convergence of probability measures $\mu_{m}$ to $M_{\sigma}(w)|d w|$. It is a well-known fact that the weak convergence of probability measures can be verified if we can check the special case $\Phi=\psi_{z}$. 
In case (FII), we define the probability measure

$$
\mu_{T}(A)=\frac{1}{2 T} \operatorname{meas}\left\{\tau^{\prime} \in[-T, T] \mid\left(L^{\prime} / L\right)\left(s+i \tau^{\prime}\right) \in A\right\}
$$

(where $A$ is any Borel subset of $\mathbb{C}$ ), and proceed similarly. The above argument was pointed out by Professor Philippe Biane and Professor Katusi Fukuyama, to whom the authors express their sincere gratitude.

\section{REFERENCES}

[1] H. Bohr, Über die Funktion $\zeta^{\prime} / \zeta(s)$, J. Reine Angew. Math. 141 (1912), 217-234.

[2] H. Bohr and B. Jessen, Über die Werteverteilung der Riemannschen Zetafunktion, I, II, Acta Math. 54 (1930), 1-35; 58 (1932), 1-55.

[3] Y. Ihara, On "M-functions" closely related to the distribution of $L^{\prime} / L$-values, Publ. Res. Inst. Math. Sci. 44 (2008), 893-954.

[4] Y. Ihara, On certain arithmetic functions $\widetilde{M}\left(s ; z_{1}, z_{2}\right)$ associated with global fields: Analytic properties, Publ. Res. Inst. Math. Sci., to appear.

[5] Y. Ihara and K. Matsumoto, On the value-distribution of $\log L$ and $L^{\prime} / L$, in "New Directions in ValueDistribution Theory of Zeta and L-Functions", Würzburg Conference (Oct 2008), R. \& J. Steuding (eds.), Shaker Verlag, 2009, pp.85-97.

[6] Y. Ihara and K. Matsumoto, On L-functions over function fields: Power-means of error-terms and distribution of $L^{\prime} / L$-values, in "Algebraic Number Theory and Related Topics 2008", H. Nakamura et al. (eds.), RIMS Kokyuroku Bessatsu B19 (2010), 221-247.

[7] Y. Ihara and K. Matsumoto, On certain mean values and the value-distribution of logarithms of Dirichlet L-functions, Quart. J. Math. (Oxford), to appear; doi:10.1093/qmath/haq002.

[8] Y. Ihara and K. Matsumoto, On $\log L$ and $L^{\prime} / L$ for $L$-functions and the associated " $M$-functions": Connections in optimal cases, Moscow Math. J., to appear.

[9] Y. Ihara, V.K. Murty and M. Shimura, On the logarithmic derivatives of Dirichlet L-functions at $s=1$, Acta Arith. 137 (2009), 253-276.

[10] B. Jessen and A. Wintner, Distribution functions and the Riemann zeta function, Trans. Amer. Math. Soc. 38 (1935), 48-88.

[11] E. R. van Kampen and A. Wintner, Convolutions of distributions on convex curves and the Riemann zeta function, Amer. J. Math. 59 (1937), 175-204.

[12] R. Kershner and A. Wintner, On the asymptotic distribution of $\zeta^{\prime} / \zeta(s)$ in the critical strip, Amer. J. Math. 59 (1937), 673-678.

[13] K. Matsumoto, Asymptotic probability measures of zeta-functions of algebraic number fields, J. Number Theory 40 (1992), 187-210.

[14] H. L. Montgomery, Topics in Multiplicative Number Theory, Lecture Notes in Math. vol.227, Springer, 1971.

RIMS, Kyoto University, Kitashirakawa-OiWAKecho, SAKyo-Ku, Kyoto, 606-8502, Japan

E-mail address: ihara@kurims.kyoto-u.ac.jp

Graduate School of Mathematics, Nagoya University, Furocho, Chikusa-ku, Nagoya, 4648602, JAPAN

E-mail address: kohjimat@math.nagoya-u.ac.jp 